\title{
Identification of CRF66_BF, a New HIV-1 Circulating Recombinant Form of South American Origin
}

\section{OPEN ACCESS}

Edited by:

Miguel Angel Martinez,

IrsiCaixa, Spain

Reviewed by:

Gonzalo Jose Bello Bentancor, Oswaldo Cruz Foundation (FIOCRUZ),

Brazil

Marcel Tongo,

Center for Research on Emerging and Re-Emerging Diseases (CREMER), Cameroon

${ }^{*}$ Correspondence: Michael M. Thomson mthomson@isciii.es

\section{Specialty section: This article was submitted to Virology, \\ a section of the journal \\ Frontiers in Microbiology}

Received: 11 September 2021 Accepted: 25 October 2021 Published: 15 November 2021

Citation:

Bacqué J, Delgado E, Benito $S$, Moreno-Lorenzo M, Montero V, Gil H,

Sánchez M, Nieto-Toboso MC, Muñoz J, Zubero-Sulibarria MZ, Ugalde E, García-Bodas E, Cañada JE, del Romero J,

Rodríguez C, Rodríguez-Avial I, Elorduy-Otazua L, Portu JJ, García-Costa J, Ocampo A, Cabrera JJ and Thomson MM (2021) Identification of CRF66_BF, a New HIV-1 Circulating Recombinant Form of South American Origin.

Front. Microbiol. 12:774386 doi: 10.3389/fmicb.2021.774386
Joan Bacqué1, Elena Delgado', Sonia Benito', María Moreno-Lorenzo', Vanessa Montero ${ }^{1}$, Horacio Gil', Mónica Sánchez', María Carmen Nieto-Toboso², Josefa Muñoz ${ }^{2}$, Miren Z. Zubero-Sulibarria², Estíbaliz Ugalde², Elena García-Bodas ${ }^{1}$, Javier E. Cañada', Jorge del Romero ${ }^{3}$, Carmen Rodríguez ${ }^{3}$, Iciar Rodríguez-Avial' ${ }^{4}$, Luis Elorduy-Otazua ${ }^{5}$, José J. Portu ${ }^{6}$, Juan García-Costa ${ }^{7}$, Antonio Ocampo $^{8}$, Jorge J. Cabrera ${ }^{8}$ and Michael M. Thomson ${ }^{1 *}$

\footnotetext{
${ }^{1}$ HIV Biology and Variability Unit, Centro Nacional de Microbiología, Instituto de Salud Carlos III, Madrid, Spain, ${ }^{2}$ Hospital Universitario de Basurto, Bilbao, Spain, ${ }^{3}$ Centro Sanitario Sandoval, IdISSC, Madrid, Spain, ${ }^{4}$ Hospital Clínico San Carlos, Madrid, Spain, ${ }^{5}$ Hospital Universitario de Cruces, Barakaldo, Spain, ${ }^{6}$ Hospital Universitario de Araba, Vitoria, Spain,

${ }^{7}$ Complejo Hospitalario Universitario de Ourense, Ourense, Spain, ${ }^{8}$ Complejo Hospitalario Universitario de Vigo, Vigo, Spain
}

Circulating recombinant forms (CRFs) are important components of the HIV-1 pandemic. Among 110 reported in the literature, 17 are BF1 intersubtype recombinant, most of which are of South American origin. Among these, all 5 identified in the Southern Cone and neighboring countries, except Brazil, derive from a common recombinant ancestor related to CRF12_BF, which circulates widely in Argentina, as deduced from coincident breakpoints and clustering in phylogenetic trees. In a HIV-1 molecular epidemiological study in Spain, we identified a phylogenetic cluster of 20 samples from 3 separate regions which were of $\mathrm{F} 1$ subsubtype, related to the Brazilian strain, in protease-reverse transcriptase (Pr-RT) and of subtype B in integrase. Remarkably, 14 individuals from this cluster (designated BF9) were Paraguayans and only 4 were native Spaniards. HIV-1 transmission was predominantly heterosexual, except for a subcluster of 6 individuals, 5 of which were men who have sex with men. Ten additional database sequences, from Argentina $(n=4)$, Spain $(n=3)$, Paraguay $(n=1)$, Brazil $(n=1)$, and Italy $(n=1)$, branched within the BF9 cluster. To determine whether it represents a new $\mathrm{CRF}$, near full-length genome (NFLG) sequences were obtained for 6 viruses from 3 Spanish regions. Bootscan analyses showed a coincident BF1 recombinant structure, with 5 breakpoints, located in p17gag, integrase, gp120, gp41-rev overlap, and nef, which was identical to that of two BF1 recombinant viruses from Paraguay previously sequenced in NFLGs. Interestingly, none of the breakpoints coincided with those of CRF12_BF. In a maximum likelihood phylogenetic tree, all 8 NFLG sequences grouped in a strongly supported clade segregating from previously identified CRFs and from the CRF12_BF "family" clade. These results allow us to identify a new HIV-1 CRF, designated CRF66_BF. Through a Bayesian coalescent analysis, the most recent common ancestor of CRF66_BF was estimated around 1984 in South America, either in Paraguay or 
Argentina. Among Pr-RT sequences obtained by us from HIV-1-infected Paraguayans living in Spain, 14 (20.9\%) of 67 were of CRF66_BF, suggesting that CRF66_BF may be one of the major HIV-1 genetic forms circulating in Paraguay. CRF66_BF is the first reported non-Brazilian South American HIV-1 CRF_BF unrelated to CRF12_BF.

Keywords: HIV-1, circulating recombinant form, molecular epidemiology, phylogeny, phylodynamics

\section{INTRODUCTION}

One of the most distinctive features of HIV-1 evolution is its high recombinogenic potential, possibly the greatest among human pathogens, which is reflected in the high frequency of unique recombinant forms (URFs), each generated in a duallyor multiply-infected individual, found wherever different genetic forms circulate in the same population (Nájera et al., 2002). Some of the HIV-1 recombinant forms have spread beyond a group of epidemiologically linked individuals, in which case they are designated circulating recombinant forms (CRFs) (Robertson et al., 2000). Currently, 110 CRFs have been reported in the literature and their number is increasing incessantly, due to both the generation of new CRFs and the identification of old previously undocumented CRFs. The proportion of CRFs in the HIV-1 pandemic has increased over time, representing around $17 \%$ infections in 2010-2015 (Hemelaar et al., 2020). Among CRFs, the most numerous are those derived from subtype $B$ and subusbtype F1, 17 of which have been identified, most of them originated in South America, derived from the F1 variant circulating in Brazil (Louwagie et al., 1994). The first CRF_BF identified in South America was CRF12_BF, which circulates widely in Argentina and Uruguay, where URFs related to CRF12_BF are frequently found (Thomson et al., 2000, 2002; Carr et al., 2001). Subsequently, 4 CRF_BFs related to CRF12_BF, as evidenced by shared breakpoints and phylogenetic clustering, were identified in the Southern Cone of South America or neighboring countries, CRF17_BF (Aulicino et al., 2012), CRF38_BF (Ruchansky et al., 2009), CRF44_BF (Delgado et al., 2010), and CRF89_BF (Delgado et al., 2021), the last three having clear country associations, with Uruguay, Chile, and Bolivia, and Peru, respectively. Due to their common ancestry, these 5 CRFs and related URFs have been proposed to constitute a "family" of recombinant viruses (Thomson and Nájera, 2005; Zhang et al., 2010; Delgado et al., 2021). By contrast, all CRF_BFs identified in Brazil are unrelated to CRF12_BF (De Sá Filho et al., 2006; Guimarães et al., 2008; Sanabani et al., 2010; Pessôa et al., 2014; Reis et al., 2017, 2019). Here we report the identification of a new CRF_BF, found mainly in Paraguayan immigrants in Spain and also identified in Paraguay and Argentina. Interestingly, unlike all South American CRF_BFs identified to date outside of Brazil, it has no relationship with CRF12_BF.

\section{MATERIALS AND METHODS}

\section{Samples}

Plasma samples from HIV-1-infected individuals were collected in 14 Spanish regions for antiretroviral drug resistance tests and for a molecular epidemiological study. The study was approved by the Committee of Research Ethics of Instituto de Salud Carlos III, Majadahonda, Madrid, Spain. The study did not require written informed consent by the study participants, as it used samples and data collected as part of routine clinical practice and patients' data were anonymized without retaining data allowing individual identification.

\section{RNA Extraction, Reverse Transcription-Polymerase Chain Reaction Amplification, and Sequencing}

An $\sim 1.4 \mathrm{~kb}$ pol fragment in protease-reverse transcriptase (Pr-RT) was amplified from plasma RNA by Reverse Transcription-Polymerase Chain Reaction (RT-PCR) followed by nested PCR as described previously (Delgado et al., 2015) and sequenced with the Sanger method using a capillary automated sequencer. Some samples were also subject to amplification and sequencing of integrase. Near full-length genome (NFLG) sequences were obtained for selected samples by RT-PCR/nested PCR amplification from plasma RNA in four overlapping segments and sequenced by the Sanger method, as described (Delgado et al., 2002; Sierra et al., 2005; Cañada et al., 2021). Newly derived sequences are deposited in GenBank under accessions MK298150, OK011530-OK011552.

\section{Phylogenetic Sequence Analyses}

Sequences were aligned with MAFFT v7 (Katoh and Standley, 2013). Initial phylogenetic trees with all Pr-RT sequences obtained by us were constructed via approximate maximum likelihood with FastTree v2.1.10 (Price et al., 2010), using the general time reversible evolutionary model with CAT approximation to account for among-site rate heterogeneity, with assessment of node support with Shimodaira-Hasegawa (SH)-like local support values (Guindon et al., 2010). A second phylogenetic tree with the Pr-RT sequences of interest and all Pr-RT sequences from the Los Alamos HIV Sequence Database (Los Alamos National Laboratory, 2021) labeled as being from F1 subsubtype or BF1 recombinant viruses, excluding those sequences identified as BF1 recombinant within Pr-RT, according to the analyses with REGA HIV1 Subtyping Tool v3 (Pineda-Peña et al., 2013), was also constructed with FastTree, as described above. Subsequent maximum likelihood (ML) trees with sequences of interest were constructed with W-IQ-Tree (Trifinopoulos et al., 2016), using the best-fit substitution model selected by ModelFinder program (Kalyaanamoorthy et al., 2017), with assessment of node support with the ultrafast bootstrap 
approximation approach (Hoang et al., 2018). Trees were visualized with MEGA v7.0 (Kumar et al., 2016) or FigTree v1.4.2 $\left(\right.$ Rambaut $^{1}$ ).

Mosaic structures were analyzed by bootscanning (Salminen et al., 1995) with SimPlot v1.3.5 (Lole et al., 1999). In these analyses, trees were constructed using the neighbor-joining method with the Kimura 2-parameter model and a window width of 400 nucleotides. Recombinant segments identified with SimPlot were further phylogenetically analyzed via ML with W-IQ-Tree. Intersubtype breakpoint locations were also determined with jpHMM (Schultz et al., 2009).

\section{Temporal and Geographical Estimations}

The time and the location of the most recent common ancestor (MRCA) of the identified CRF was estimated using Pr-RT sequences with the Bayesian Markov chain Monte Carlo (MCMC) coalescent method implemented in BEAST v1.10.4 (Suchard et al., 2018), using a discrete trait approach. Prior to the BEAST analysis, the existence of temporal signal in the dataset was assessed with TempEst v1.5.3 (Rambaut et al., 2016), which determines the correlation of genetic divergence among sequences (measured as root-to-tip distance) with time. The BEAST analysis was performed using the SRD06 codon-based evolutionary model (with two codon position partitions: $1 \mathrm{st}+2^{\text {nd }}$, and 3rd) (Shapiro et al., 2006). A uniform prior distribution $\left(2 \times 10^{-4}-2 \times 10^{-2}\right.$ subs/site/year) was used for the substitution rate. We also specified an uncorrelated lognormal relaxed clock and a Bayesian SkyGrid coalescent tree prior (Gill et al., 2013). In the SkyGrid analysis, the number of grid points was set at 50 and the time at last transition point at 60 years. The MCMCs were run for 50 million generations. We performed runs in duplicate, combining the posterior tree files with LogCombiner v1.10.4. Mixing and convergence were checked with Tracer v1.6, ensuring that effective sample size values of all parameters were $>200$. The posterior distribution of trees was summarized in a maximum clade credibility (MCC) tree with TreeAnnotator v1.10.4, after removal a $10 \%$ burn-in. MCC trees were visualized with FigTree. Parameter uncertainty was summarized in 95\% highest posterior density (HPD) intervals.

\section{RESULTS}

\section{Identification of a BF Intersubtype Recombinant Cluster and Epidemiological Associations}

In a molecular epidemiology study on HIV-1 in Spain we identified a cluster of 20 viruses of F1 subsubtype in Pr-RT, that in integrase, sequenced in 4 samples, were of subtype B, which was designated BF9. Inclusion in the phylogenetic analyses of $\mathrm{Pr}-$ RT sequences from all viruses in the Los Alamos HIV Sequence Database (Los Alamos National Laboratory, 2021) classified as being of $\mathrm{F} 1$ subsubtype or BF1 recombinant, excluding those sequences that were $\mathrm{BF} 1$ recombinant within $\mathrm{Pr}-\mathrm{RT}$, according to REGA HIV-1 Subtyping Tool, identified 10 additional viruses

${ }^{1}$ http://tree.bio.ed.ac.uk/software/figtree/ belonging to BF9, from Argentina $(n=4)$, Spain $(n=3)$, Paraguay $(n=1)$, Brazil $(n=1)$, and Italy $(n=1)$ (Figure 1 and Supplementary Figure 1). Pr-RT sequences of the BF9 cluster were most closely related to F1 viruses of the Brazilian variant (Figure 1). Epidemiological data of the 20 samples of the BF9 cluster from Spain processed by us are shown in Table 1. Remarkably, 14 individuals were from Paraguay [with the remaining 6 being from Spain $(n=4)$, Argentina, and Equatorial Guinea] and all 3 other database sequences from samples collected in Spain were from Latin Americans, one each from Paraguay, Argentina, and an unspecified country. Transmission was predominantly heterosexual, but 7 were men who have sex with men (MSM), the sequences of 5 of whom branched in a subcluster (Figure 1).

\section{Analyses of Near Full-Length Genome Sequences and Identification of a New Circulating Recombinant Form}

To determine whether viruses from the BF9 cluster represent a new CRF, we obtained NFLG sequences from 6 samples from 3 Spanish regions and analyzed their mosaic structures by bootscanning. Two additional NFLG sequences of BF recombinant viruses from databases were also analyzed by bootscanning, both from Paraguay: 02PY_PSP0094, that branched in the BF9 cluster in Pr-RT, and 02PY_PSP0093, that showed high similarity to NFLGs of the BF9 cluster in BLAST searches of the Los Alamos database. All 8 analyzed genomes showed coincident mosaic structures, with 5 breakpoints, located in p17gag, integrase, gp120, gp41-rev overlap, and nef (Figure 2). Breakpoints were more precisely located using the midpoint of B-F1 transitions, according to the positions where $75 \%$ consensuses of subtype B and the F1 Brazilian strain genomes differ, in HXB2 positions 950, 4327, 6486, 8498, and 9161. Breakpoint locations were also determined with jpHMM (Supplementary Table), which also found 5 breakpoints for each virus in intervals overlapping those of the other analyzed viruses and the $75 \%$ consensus B-F1 transition intervals in all cases except the breakpoint interval in nef of MD497796, that did not overlap the consensus B-F1 transition interval, and that in p17 gag of PV106451, that was not detected by jpHMM. ML phylogenetic trees constructed with each interbreakpoint fragment confirmed the subtype assignation determined with bootscanning (Figure 3).

In an ML tree constructed with the 7 NFLG genomes of the BF9 cluster and 02PY_PSP0093, all 8 genomes grouped in a strongly supported clade segregating away from all other CRF_BFs and of the clade formed by the 5 CRF_BFs of the CRF12_BF family (Figure 4). It should be pointed out that 02PY_PSP0093 did not branch in the BF9 cluster in the tree of Pr-RT (Supplementary Figure 1), which suggests that the Pr-RT segment of this virus could derive from secondary recombination with an F1 strain different from the parental F1 strain of all other BF9 viruses.

These results, therefore, allow to define a new CRF, which was designated CRF66_BF, whose mosaic structure is shown in Figure 5. 


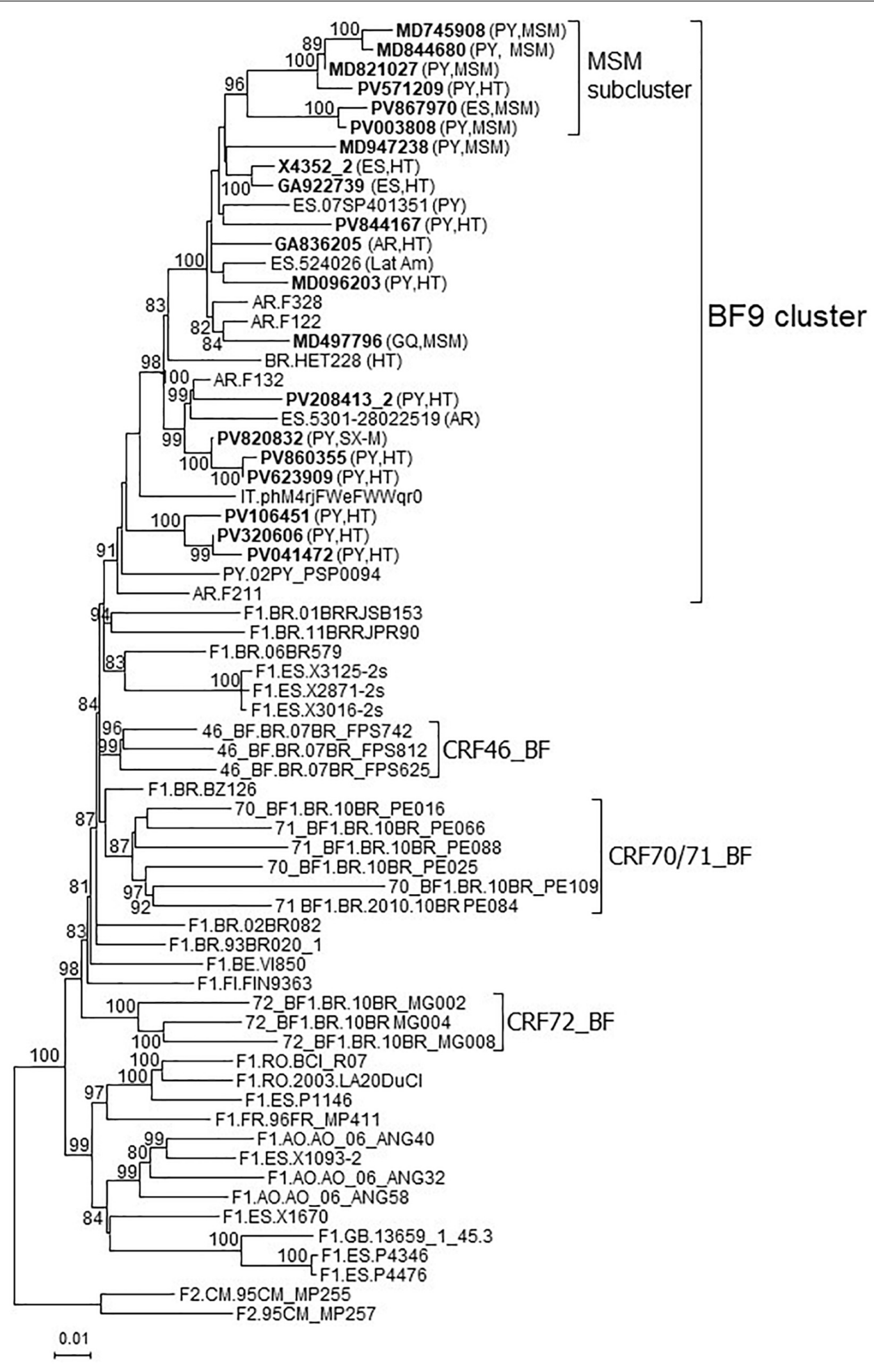

FIGURE 1 | Maximum likelihood tree of Pr-RT sequences of BF9 cluster constructed with IQ-Tree. Sequences used in this analysis were those from the BF9 cluster shown in the tree of Supplementary Figure 1, constructed with FastTree, plus sequences of F1 subsubtype from different countries and of CRF_BFs that are of nonrecombinant F1 subsubtype in Pr-RT, plus two F2 sequences used as outgroups. Names of sequences obtained by us, all collected in Spain, are in bold type. In database sequences, the country of sample collection is indicated before the virus name with the 2-letter ISO country code. After the names of viruses of the BF9 cluster, the 2-letter ISO code of country of origin of the patient and/or the transmission route, when known, are shown in parentheses. Only ultrafast bootstrap values 280\% are shown. PY: Paraguay; AR: Argentina; ES: Spain; BR: Brazil; IT: Italy; GQ: Equatorial Guinea; Lat Am: Latin America (unknown country); MSM: man who has sex with men; HT: heterosexual; SX-M: male with unspecified sexual acquisition of HIV-1. 
TABLE 1 | Epidemiological data of patients and GenBank accessions of sequences.

\begin{tabular}{|c|c|c|c|c|c|c|c|c|}
\hline Sample ID & $\begin{array}{l}\text { City of sample } \\
\text { collection }\end{array}$ & $\begin{array}{l}\text { Region of sample } \\
\text { collection }\end{array}$ & $\begin{array}{l}\text { Year of sample } \\
\text { collection }\end{array}$ & $\begin{array}{l}\text { Year of HIV } \\
\text { diagnosis }\end{array}$ & Gender & $\begin{array}{l}\text { Transmission } \\
\text { route* }^{*}\end{array}$ & Country of origin & GenBank accessions \\
\hline X4352_2 & Vigo & Galicia & 2017 & 2017 & M & $\mathrm{HT}$ & Spain & MK298150 (NFLG) \\
\hline GA836205 & Vigo & Galicia & 2020 & 2020 & M & HT & Argentina & $\begin{array}{l}\text { OK011531 (Pr-RT) } \\
\text { OK011530 (integrase) }\end{array}$ \\
\hline GA922739 & Ourense & Galicia & 2018 & 2017 & M & $\mathrm{HT}$ & Spain & OK011532 (NFLG) \\
\hline MD096203 & Madrid & Madrid & 2017 & 2011 & $\mathrm{~F}$ & HT & Paraguay & $\begin{array}{l}\text { OK011534 (Pr-RT) } \\
\text { OK011533 (integrase) }\end{array}$ \\
\hline MD497796 & Madrid & Madrid & 2017 & 2017 & M & MSM & Equatorial Guinea & OK011534 (NFLG) \\
\hline MD745908 & Madrid & Madrid & 2019 & 2019 & M & MSM & Spain & OK011536 (Pr-RT) \\
\hline MD821027 & Madrid & Madrid & 2018 & 2018 & M & MSM & Paraguay & OK011537 (Pr-RT) \\
\hline MD844680 & Madrid & Madrid & 2020 & 2020 & M & MSM & Paraguay & OK011538 (Pr-RT) \\
\hline MD947238 & Madrid & Madrid & 2018 & 2016 & M & MSM & Paraguay & OK011539 (Pr-RT) \\
\hline PV003808 & Bilbao & Basque Country & 2020 & 2020 & M & MSM & Paraguay & $\begin{array}{l}\text { OK011541 (Pr-RT) } \\
\text { OK011540 (integrase) }\end{array}$ \\
\hline PV041472 & Bilbao & Basque Country & 2014 & 2014 & $\mathrm{~F}$ & HT & Paraguay & OK011542 (Pr-RT) \\
\hline PV106451 & Bilbao & Basque Country & 2010 & 2010 & $\mathrm{~F}$ & HT & Paraguay & OK011543 (NFLG) \\
\hline PV208413_2 & Bilbao & Basque Country & 2009 & 2009 & M & HT & Paraguay & OK011544 (Pr-RT) \\
\hline PV320606 & Bilbao & Basque Country & 2014 & 2014 & M & HT & Paraguay & OK011545 (Pr-RT) \\
\hline PV571209 & Bilbao & Basque Country & 2013 & 2013 & M & HT & Paraguay & OK011546 (Pr-RT) \\
\hline PV623909 & Bilbao & Basque Country & 2011 & 2011 & $\mathrm{~F}$ & $\mathrm{HT}$ & Paraguay & OK011547 (NFLG) \\
\hline PV820832 & Bilbao & Basque Country & 2008 & 2008 & M & Sexual & Paraguay & OK011548 (Pr-RT) \\
\hline PV844167 & Vitoria & Basque Country & 2016 & 2016 & M & $\mathrm{HT}$ & Paraguay & OK011549 (NFLG) \\
\hline PV860355 & Bilbao & Basque Country & 2011 & 2011 & M & HT & Paraguay & OK011550 (Pr-RT) \\
\hline PV867970 & Bilbao & Basque Country & 2020 & 2020 & M & MSM & Spain & $\begin{array}{l}\text { OK011552 (Pr-RT) } \\
\text { OK011551 (integrase) }\end{array}$ \\
\hline
\end{tabular}

*HT: heterosexual; MSM: man who has sex with men. 

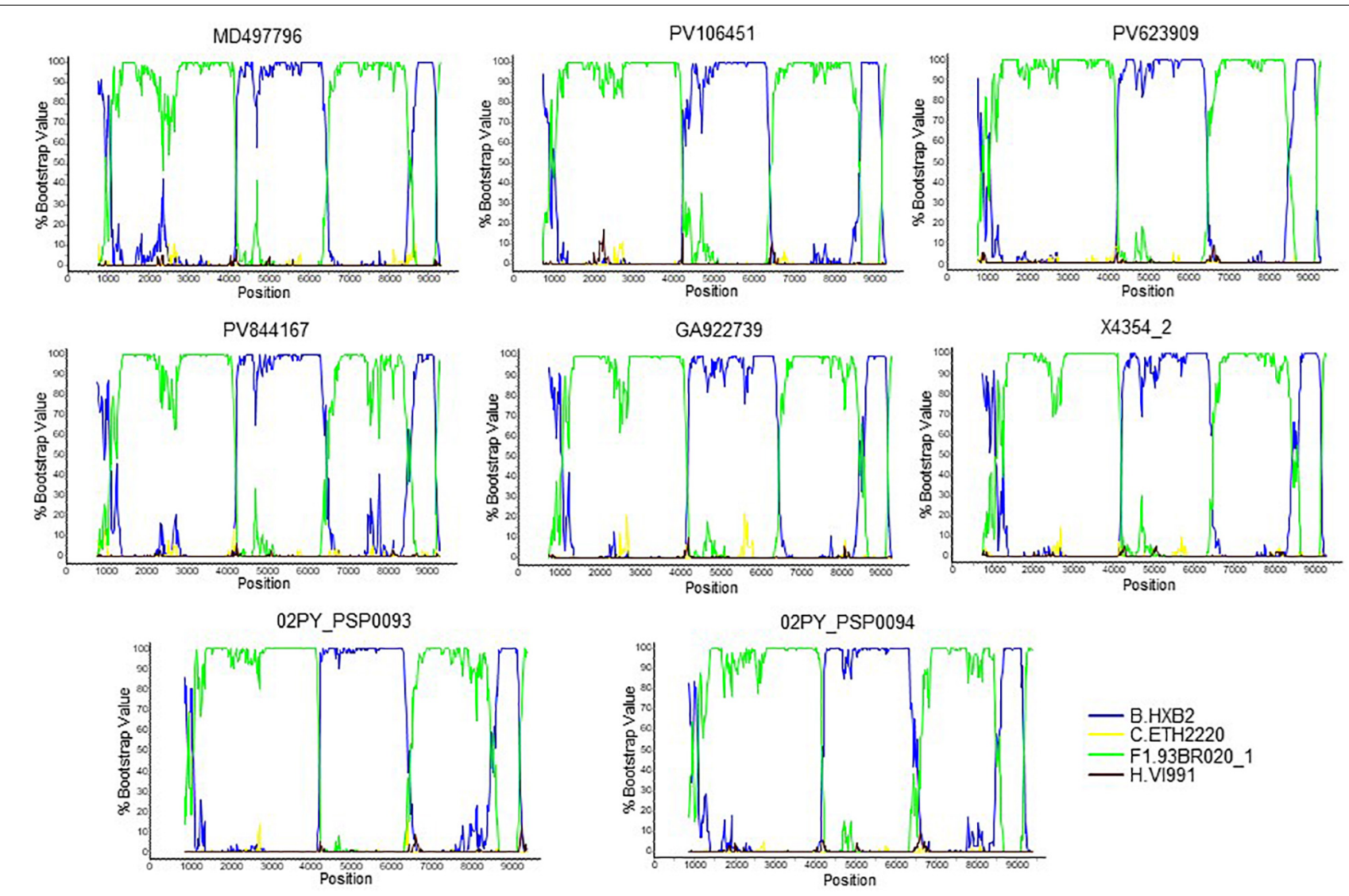

FIGURE 2 | Bootscan analyses of 6 NFLG sequences of viruses of the BF9 cluster obtained by us and of two BF1 database NFLG sequences from Paraguay, 02PY_PSP0093 and 02PY_PSP0094. The horizontal axis represents the position in the HXB2 genome of the midpoint of a 400 nt window moving in 20 nt increments and the vertical axis represents bootstrap values supporting clustering with subtype reference sequences.

\section{Prevalence of CRF66_BF Among HIV-1-Infected Paraguayans Residing in Spain and Among Sequences From Samples Collected in Paraguay Deposited at the HIV-1 Sequence Database}

Among $67 \mathrm{HIV}$-1-infected Paraguayans residing in Spain studied by us, CRF66_BF was the most common non-subtype B genetic form, representing $20.9 \%$ (14 of 67 ) of total infections, $48.3 \%$ (14 of 29) of non-subtype B infections, and 60.1\% (14 of 23) of F1/BF1 infections.

At the Los Alamos HIV Sequence Database, there are HIV1 sequences from samples collected in Paraguay from only 23 individuals, of which 12 are NFLG sequences from samples collected in 2002 or 2003 and 11 are env V3 region sequences from samples with unknown collection years. In phylogenetic analyses, 2 of 12 NFLG and 1 of $11 \mathrm{~V} 3$ sequences [combined, 3 (13\%) of 23 viruses] branched in the clade formed by CRF66_BF viruses (Supplementary Figure 2). However, due to the short length of the Paraguayan V3 sequences, the reliability of the tree of this segment for identifying CRF66_BF viruses is uncertain, since several CRF_BF and the subtype $G$ references branched apart from other references of the same genetic form, and one CRF72_BF reference branched within the CRF66_BF clade.

\section{Temporal and Geographical Estimations of CRF66_BF Origin}

To estimate the time and place of origin of CRF66_BF, Pr-RT sequences where analyzed with a Bayesian coalescent method with BEAST 1.10.4. Prior to this analysis, TempEst analysis determined that there was an adequate temporal signal in the dataset $\left(r^{2}=0.5871\right)$. In the BEAST analysis, for the sequences corresponding to South American individuals residing in Spain, the assigned location trait was their country of origin, rather than their place of residence. This was done because most individuals harboring CRF66_BF identified in Spain were of South American origin (mostly from Paraguay) and because we found no definitive evidence of the local circulation of CRF66_BF in Spain, as reflected in clusters mainly comprising Spanish individuals. Therefore, we assumed that South Americans harboring CRF66_BF viruses had acquired HIV-1 in their countries of origin. In this analysis, the substitution rate was estimated at $1.987 \times 10^{-3}$ subs/site/year (95\% HPD, $8.885 \times 10^{-4}-3.282 \times 10^{-3}$ subs/site/year) and the time of the MRCA of CRF66_BF was estimated around 


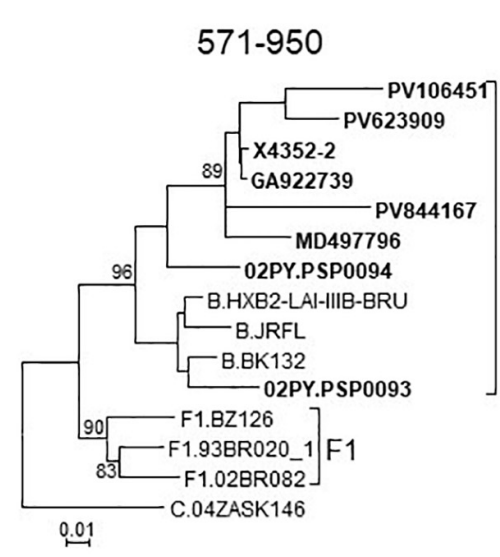

6486-8497

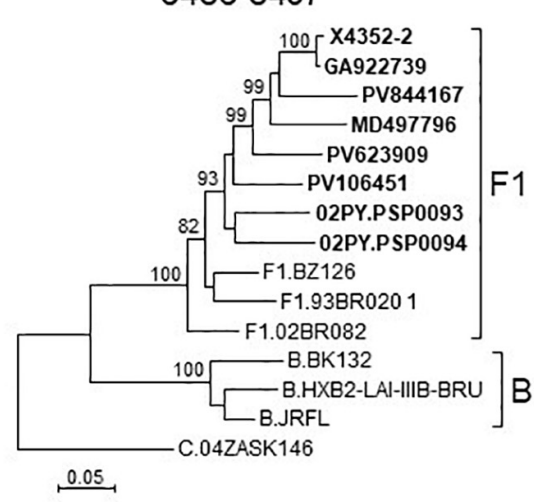

$951-4326$

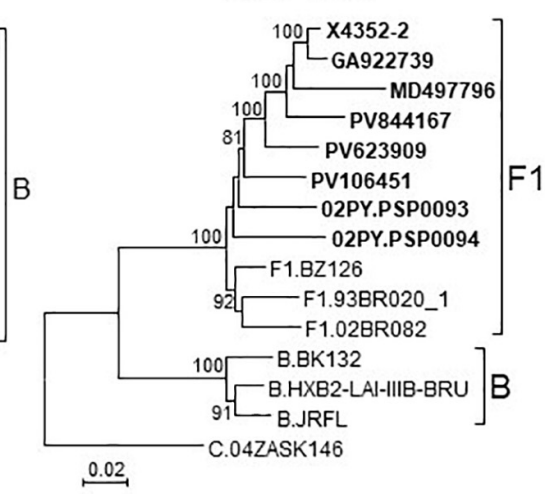

8498-9161
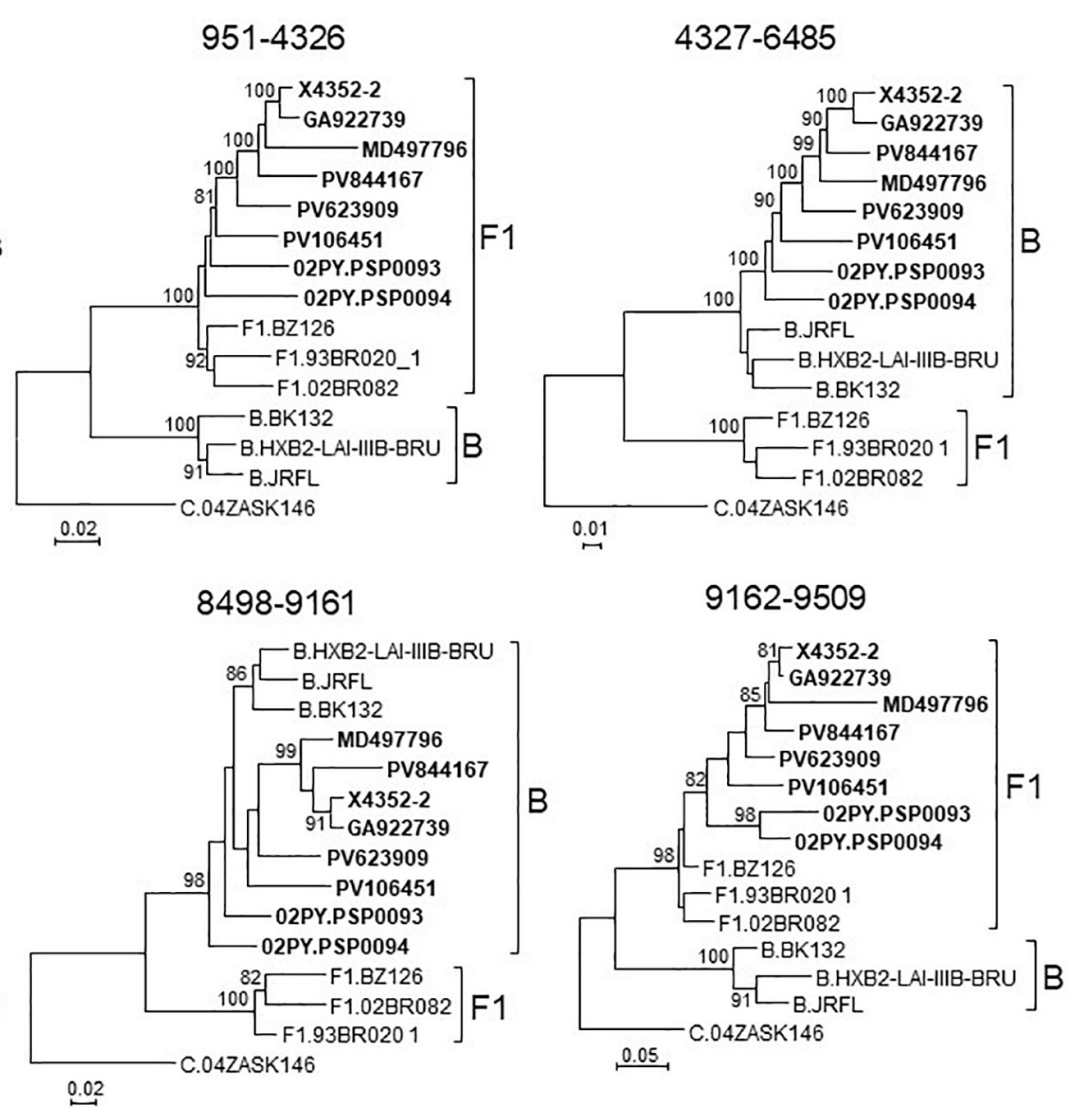

FIGURE 3 | Phylogenetic trees of interbreakpoint genome segments of the BF recombinant viruses analyzed by bootscanning, constructed with IQ-Tree. HXB2 positions delimiting the analyzed segments are indicated on top of the trees. Sequence names of BF viruses are in bold type. Names of subtype references are preceded by the corresponding subtype name. Only ultrafast bootstrap values $\geq 80 \%$ are shown.

1984 (95\% HPD, 1970-1996), with its most probable location in Paraguay $(\mathrm{PP}=0.76)$, with Argentina in second place $(\mathrm{PP}=0.22)$ (Figure 6). Considering the possibility that local subclusters each found in one city could represent local transmissions, we performed a second analysis in which we assigned the country of location of the most recent diagnoses of such clusters to Spain, irrespective of the countries of origin of the individuals. In this analysis, Paraguay was also estimated as the most probable location of the MRCA of CRF66_BF, although with a lower support $(\mathrm{PP}=0.55)$, and the support for Argentina increased to a $\mathrm{PP}=0.42$ (Supplementary Figure 3).

\section{DISCUSSION}

The results of this study allow to define a new HIV-1 CRF, designated CRF66_BF, which is the $18^{\text {th }}$ reported CRF derived from subtypes B and F. Samples harboring CRF66_BF were collected in 5 countries, in South America (Argentina, Paraguay, and Brazil) and Western Europe (Spain and Italy), with a majority collected in Spain. However, of samples collected in Spain, a great majority were from Paraguayan individuals. Bayesian coalescent analyses (performed with the assumption that South American individuals living in Spain harboring CRF66_BF viruses had acquired them in their countries of origin), pointed to a most probable origin of CRF66_BF in Paraguay $(\mathrm{PP}=0.76)$, with Argentina being the second most probable location $(\mathrm{PP}=0.22)$. When the analysis was performed assigning the most recently diagnosed samples of clusters found in a single Spanish city to Spain as the location trait, irrespective of the country of origin of the individual, the PPs for a MRCA in Paraguay or Argentina were not very different ( 0.55 vs. 0.42 , respectively). Therefore, the results point to a South American origin of CRF66_BF, either in Paraguay or Argentina, without a definitive support for either country. However, given the great predominance of Paraguayans among CRF66_BF-infected individuals living in Spain, we cannot rule out that the same could happen in Argentina, where Paraguayans represent the largest immigrant national group (Instituto Nacional de Estadísticas y Censos, República Argentina, 2021). If this was the case, and information on country of origin of the sampled individuals living in Argentina was included in the analyses, it is possible that the support for a root of the CRF66_BF tree in Paraguay would increase.

The estimated date of origin of CRF66_BF around 1984 is consistent with the published estimated origin of the Brazilian F1 strain (around 1977) (Bello et al., 2007) and is similar to those 


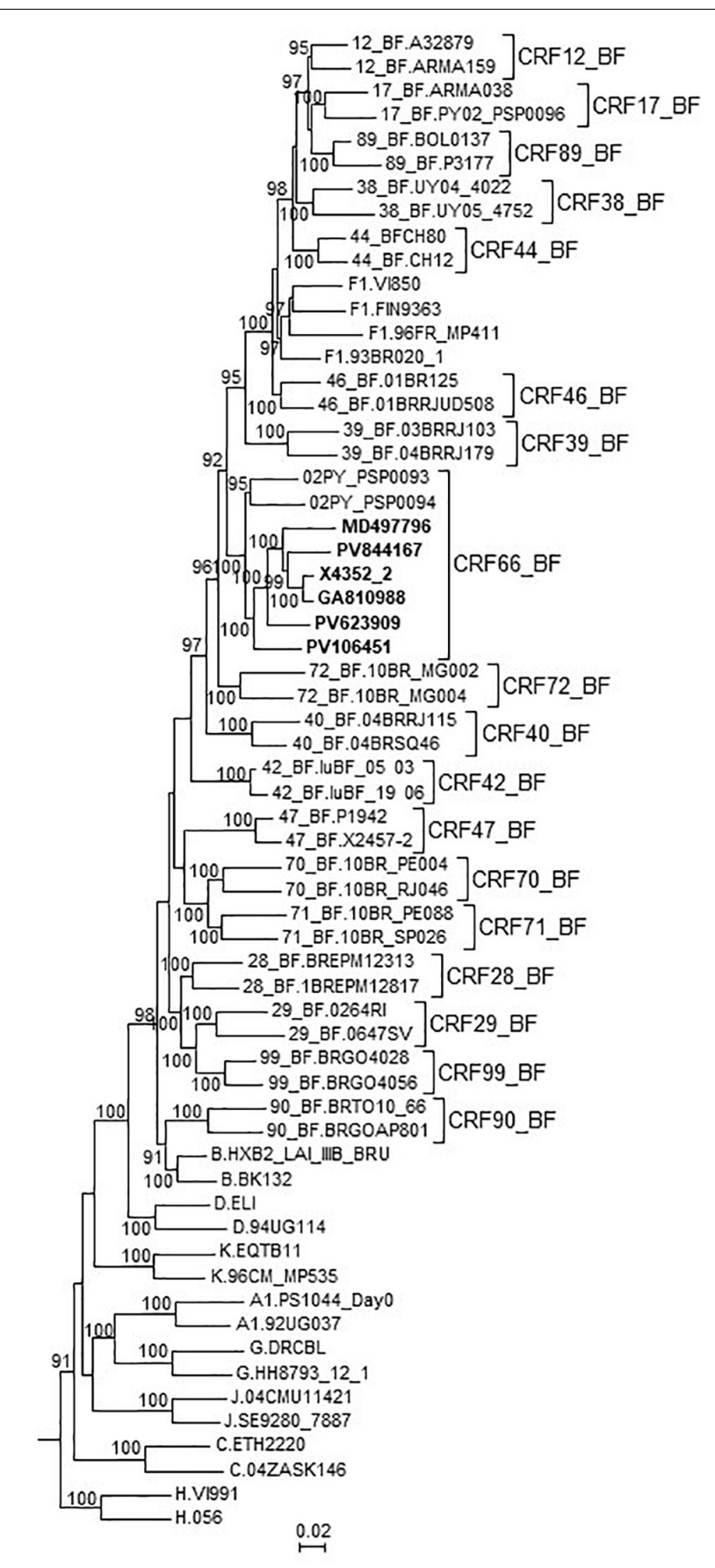

FIGURE 4 | Maximum likelihood tree of NFLG sequences of viruses of the BF9 cluster and PY02_PSP0094, constructed with IQ-Tree. References of all published CRF_BFs and of HIV-1 subtypes are also included in the analysis. The tree is rooted with SIVcpz virus MB66. Names of sequences obtained by us are in bold type. In reference sequences, the subtype or CRF is indicated before the virus name. Only ultrafast bootstrap values $\geq 90 \%$ are shown.

of other South American CRF_BFs (CRF12, CRF28/29, CRF38, CRF89, and CRF90) reported in the literature (Bello et al., 2010; Ristic et al., 2011; Reis et al., 2017; Delgado et al., 2021), although younger than some other estimates for CRF12_BF in the 1970s
(Dilernia et al., 2011; Delgado et al., 2021) and older than the estimate for CRF99_BF, around 1993 (Reis et al., 2017).

Among HIV-1-infected Paraguayans residing in Spain studied by us, there was relatively high prevalence (21\%) of CRF66_BF infections, which suggests that CRF66_BF could be one of the major HIV-1 genetic forms circulating in Paraguay. A better knowledge of the current prevalence of CRF66_BF in Paraguay would require sequencing a representative sample of recent HIV1 diagnoses in the country. However, HIV-1 sequences from only 23 patients sampled in Paraguay are available at the Los Alamos HIV Sequence database, and the most recent molecular epidemiological study published to date involves the analysis of sequences from 55 samples collected 18 to 19 years ago (Aguayo et al., 2008), which are not available in public databases.

Notably, CRF66_BF, unlike all other non-Brazilian CRF_BFs identified to date in South America (CRF12_BF, CRF17_BF, CRF38_BF, CRF44_BF, and CRF89_BF, all circulating in the Southern Cone or neighboring countries), is unrelated to CRF12_BF, as deduced from the lack of breakpoint coincidence and of phylogenetic clustering with CRF12_BF. This implies that CRF66_BF originated independently from viruses of the CRF12_BF family, with a presumable ancestry in Brazil, where $\mathrm{B}$ and $\mathrm{F} 1$ viruses are circulating (Louwagie et al., 1994).

CRF66_BF is the 5th CRF of South American ancestry originally identified in Western Europe [after CRF42_BF (Struck et al., 2015), CRF47_BF (Fernández-García et al., 2010), CRF60_BC (Simonetti et al., 2014), and CRF89_BF (Delgado et al., 2021)], which, together with the reported propagation of HIV-1 variants of South America origin among the European population (de Oliveira et al., 2010; Collaço Verás et al., 2012; Thomson et al., 2012; Lai et al., 2014; Carvalho et al., 2015; Delgado et al., 2015; Fabeni et al., 2015, 2020; Vinken et al., 2019), points to an increasing relationship between the HIV-1 epidemics in both continents. This reflects migratory fluxes, most notably in Spain, where around 2.5 million South Americans live, representing nearly $40 \%$ of the migrant population (Instituto Nacional de Estadística, 2021a), and immigration from South America has increased greatly in recent years (Instituto Nacional de Estadística, 2021b) (Supplementary Figure 4). Considering the large and increasing South American immigrant population in Europe and the relative scarcity of HIV-1 sequences available in public databases from some South American countries (such as Colombia, Guyana, Ecuador, Bolivia, Paraguay, Chile, and Uruguay) (Supplementary Figure 5), studies on HIV-1 genetic diversity and molecular epidemiology among South American immigrants living in Europe could provide novel insights into the HIV-1 epidemics in their countries of origin, although the acquisition of some HIV-1 infections in their European countries of residence, reflected in branching in European clusters, should be taken into account (Osorno et al., 11th Conference on HIV Science, abstract PEC252, 18-21 July 20212), as well as on the diffusion of South American HIV-1 variants in Europe.

It is interesting to note that although transmission of CRF66_BF is predominantly via heterosexual contact, most individuals in a cluster are MSM (Figure 1), which suggests

\footnotetext{
${ }^{2}$ https://ias2021.org/wp-content/uploads/2021/07/IAS2021_Abstracts_web.pdf
} 


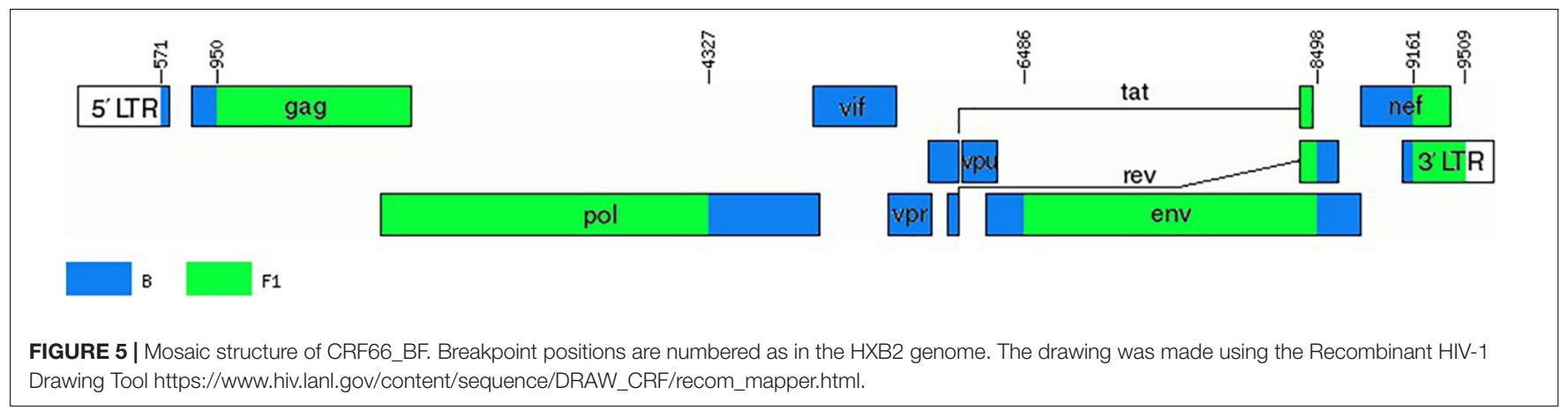
Drawing Tool https://www.hiv.lanl.gov/content/sequence/DRAW_CRF/recom_mapper.html.

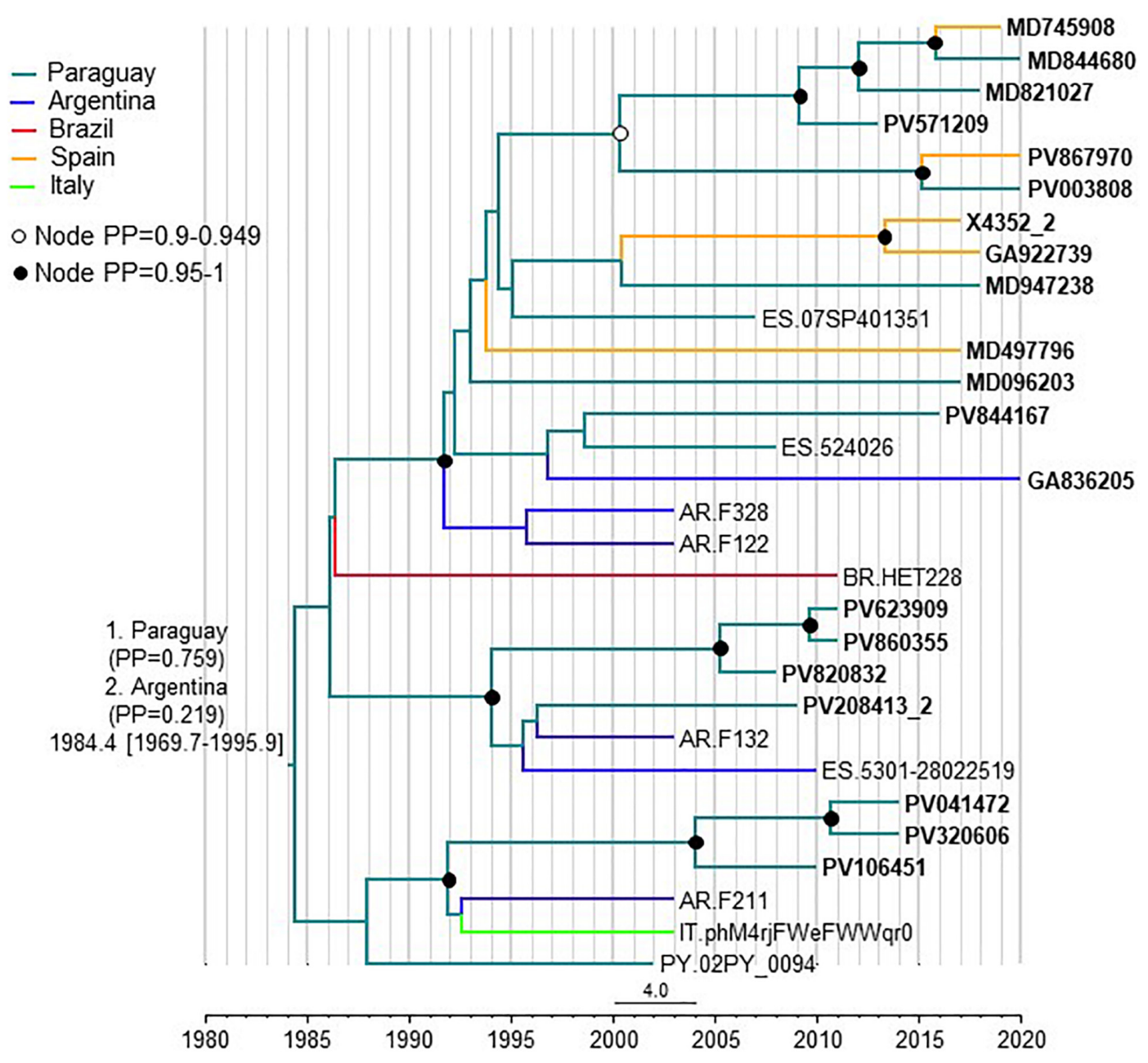

FIGURE 6 | Maximum clade credibility tree of CRF66_BF Pr-RT sequences. Branch colors indicate, for terminal branches, country of sample collection or, for South American individuals residing in Spain, of origin of the individual, which was used as location trait (see Methods), and for internal branches, the most probable location country of the subtending node, according to the legend on the upper left. For database sequence 524026, from a sample collected in Spain, location was assigned to Paraguay as the most probably country of origin, although the only available information in the GenBank entry is that the individual was from Latin America, because 15 (88.2\%) of 17 Latin Americans with CRF66_BF sampled in Spain were from Paraguay. Nodes supported by PP $\geq 0.95$ and PP 0.9-0.949 are indicated with filled and unfilled circles, respectively. The two most probable countries at the root of the tree are indicated, together with the PPs supporting each location and the time of the MRCA (mean value, with 95\% HPD interval in brackets).

diffusion from a heterosexual to a MSM network. HIV-1 propagation between heterosexual and MSM networks has also been reported for CRF89_BF (Delgado et al., 2021) and for a large CRF02_AG cluster in Spain (Delgado et al., 2019), although in the latter case the direction of propagation was from MSM to heterosexuals. 
One of the essential tasks of Biology is naming and classifying organisms. In this work, we have accomplished this task by identifying a new HIV-1 circulating recombinant form, derived from subtypes B and F1, named CRF66_BF. CRF66_BF most likely originated in South America, either in Paraguay or Argentina, and, unlike all non-Brazilian South American CRFs identified to date, is unrelated to CRF12_BF. The identification and genetic characterization of HIV-1 variants is the first and necessary step for molecular epidemiological studies examining their geographic dissemination, growth dynamics, and epidemiological associations, as well as for analyzing their biological properties, such as pathogenic and transmissibility potentials, response to antiretroviral therapies, and susceptibility to immune responses inducible by vaccines. Such studies on CRF66_BF and other South American CRFs may be the subject of future work.

\section{DATA AVAILABILITY STATEMENT}

The names of the repository and accession numbers can be found in the article (Materials and Methods and Table 1).

\section{ETHICS STATEMENT}

The studies involving human participants were reviewed and approved by Committe of Research Ethics, Instituto de Salud Carlos III, Madrid, Spain. Written informed consent for participation was not required for this study in accordance with the national legislation and the institutional requirements.

\section{AUTHOR CONTRIBUTIONS}

MT and ED conceived the study and supervised the experimental work. JB, MT, and ED processed sequences and performed

\section{REFERENCES}

Aguayo, N., Laguna-Torres, V. A., Villafane, M., Barboza, A., Sosa, L., Chauca, G., et al. (2008). Epidemiological and molecular characteristics of HIV-1 infection among female commercial sex workers, men who have sex with men and people living with AIDS in Paraguay. Rev. Soc. Bras. Med. Trop. 41, 225-231. doi: 10.1590/s0037-86822008000300001

Aulicino, P. C., Gómez-Carrillo, M., Bello, G., Rocco, C., Mangano, A., Carr, J., et al. (2012). Characterization of full-length HIV-1 CRF17_BF genomes and comparison to the prototype CRF12_BF strains. Infect. Genet. Evol. 12, 443-447.

Bello, G., Aulicino, P. C., Ruchansky, D., Guimarães, M. L., López-Galíndez, C., Casado, C., et al. (2010). Phylodynamics of HIV-1 circulating recombinant forms 12_BF and 38_BF in Argentina and Uruguay. Retrovirology 7:22. doi: 10.1186/1742-4690-7-22

Bello, G., Eyer-Silva, W. A., Couto-Fernandez, J. C., Guimarães, M. L., ChequerFernandez, S. L., Teixeira, S. L., et al. (2007). Demographic history of HIV-1 subtypes B and F in Brazil. Infect. Genet. Evol. 7, 263-270.

Cañada, J. E., Delgado, E., Gil, H., Sánchez, M., Benito, S., García-Bodas, E., et al. (2021). Identification of a new HIV-1 BC intersubtype circulating recombinant form (CRF108_BC) in Spain. Viruses 13:93. doi: 10.3390/v13010093 phylogenetic analyses. MT performed phylodynamic analyses. HG performed data curation. JB, SB, MM-L, VM, MS, EG-B, and JEC performed experimental work. MN-T, JM, MZ-S, EU, JR, CR, IR-A, LE-O, JP, JG-C, AO, and JJC obtained samples and epidemiological data from patients. MT, $\mathrm{ED}$, and $\mathrm{HG}$ wrote the manuscript. All authors read and approved the manuscript.

\section{FUNDING}

This work was funded through Acción Estratégica en Salud Intramural (AESI), Instituto de Salud Carlos III, projects PI16CIII/00033 and PI19CIII/00042; Red de Investigación en SIDA (RIS), Instituto de Salud Carlos III, Subdirección General de Evaluación y Fondo Europeo de Desarrollo Regional (FEDER), Plan Nacional I+D+I, project RD16ISCIII/0002/0004; and scientific agreements with Consellería de Sanidade, Government of Galicia (MVI 1004/16) and Osakidetza-Servicio Vasco de Salud, Government of Basque Country (MVI 1001/16).

\section{ACKNOWLEDGMENTS}

We thank José Antonio Taboada, from Consellería de Sanidade, Xunta de Galicia, and Daniel Zulaika, from Osakidetza-Servicio Vasco de Salud, for their support of this study, and the personnel at the Genomic Unit, Instituto de Salud Carlos III, for technical assistance in sequencing.

\section{SUPPLEMENTARY MATERIAL}

The Supplementary Material for this article can be found online at: https://www.frontiersin.org/articles/10.3389/fmicb. 2021.774386/full\#supplementary-material

Carr, J. K., Avila, M., Gomez Carrillo, M., Salomon, H., Hierholzer, J., Watanaveeradej, V., et al. (2001). Diverse BF recombinants have spread widely since the introduction of HIV-1 into South America. AIDS 15, F41-F47. doi: 10.1097/00002030-200110190-00002

Carvalho, A., Costa, P., Triunfante, V., Branca, F., Rodrigues, F., Santos, C. L., et al. (2015). Analysis of a local HIV-1 epidemic in Portugal highlights established transmission of non-B and non-G subtypes. J. Clin. Microbiol. 53, 1506-1514. doi: 10.1128/JCM.03611-14

Collaço Verás, N. M., Gray, R. R., de Macedo Brígido, L. F., Rodrigues, R., and Salemi, M. (2012). High-resolution phylogenetics and phylogeography of human immunodeficiency virus type 1 subtype C epidemic in South America. J. Gen. Virol. 92, 1698-1709. doi: 10.1099/vir.0.028951-0

de Oliveira, T., Pillay, D., and Gifford, R. J. (2010). The HIV-1 subtype C epidemic in South America is linked to the United Kingdom. PLoS One 5:e9311. doi: 10.1371/journal.pone.0009311

De Sá Filho, D. J., Sucupira, M. C., Caseiro, M. M., Sabino, E. C., Diaz, R. S., and Janini, L. M. (2006). Identification of two HIV type 1 circulating recombinant forms in Brazil. AIDS Res. Hum. Retroviruses 22, 1-13. doi: 10.1089/aid.2006. 22.1

Delgado, E., Benito, S., Montero, V., Cuevas, M. T., Fernández-García, A., SánchezMartínez, M., et al. (2019). Diverse large HIV-1 non-subtype B clusters are 
spreading among men who have sex with men in Spain. Front. Microbiol. 10:655. doi: 10.3389/fmicb.2019.00655

Delgado, E., Cuevas, M. T., Domínguez, F., Vega, Y., Cabello, M., FernándezGarcía, A., et al. (2015). Phylogeny and phylogeography of a recent HIV-1 subtype F outbreak among men who have sex with men in Spain deriving from a cluster with a wide geographic circulation in Western Europe. PLoS One 10:e0143325. doi: 10.1371/journal.pone.0143325

Delgado, E., Fernández-García, A., Pérez-Losada, M., Moreno-Lorenzo, M., Fernández-Miranda, I., Benito, S., et al. (2021). Identification of CRF89_BF, a new member of an HIV-1 circulating BF intersubtype recombinant form family widely spread in South America. Sci. Rep. 11:11442. doi: 10.1038/s41598-02190023-x

Delgado, E., Ríos, M., Fernández, J., Pérez-Alvarez, L., Nájera, R., and Thomson, M. M. (2010). Identification of a new HIV type 1 BF intersubtype circulating recombinant form (CRF44_BF) in Chile. AIDS Res. Hum. Retroviruses 26, 821-826. doi: 10.1089/aid.2010.0006

Delgado, E., Thomson, M. M., Villahermosa, M. L., Sierra, M., Ocampo, A., Miralles, C., et al. (2002). Identification of a newly characterized HIV-1 BG intersubtype circulating recombinant form in Galicia, Spain, which exhibits a pseudotype-like virion structure. J. Acquir. Immune Defic. Syndr. 29, 536-543. doi: 10.1097/00126334-200204150-00016

Dilernia, D. A., Jones, L. R., Pando, M. A., Rabinovich, R. D., Damilano, G. D., Turk, G., et al. (2011). Analysis of HIV type 1 BF recombinant sequences from South America dates the origin of CRF12_BF to a recombination event in the 1970s. AIDS Res. Hum. Retroviruses 27, 569-578. doi: 10.1089/AID.2010.0118

Fabeni, L., Alteri, C., Orchi, N., Gori, C., Bertoli, A., Forbici, F., et al. (2015). Recent transmission clustering of HIV-1 C and CRF17_BF strains characterized by NNRTI-related mutations among newly diagnosed men in Central Italy. PLoS One 10:e0135325. doi: 10.1371/journal.pone.0135325

Fabeni, L., Santoro, M. M., Lorenzini, P., Rusconi, S., Gianotti, N., Costantini, A., et al. (2020). Evaluation of HIV transmission clusters among natives and foreigners living in Italy. Viruses 12:791. doi: 10.3390/v12080791

Fernández-García, A., Pérez-Alvarez, L., Cuevas, M. T., Delgado, E., Muñoz-Nieto, M., Cilla, G., et al. (2010). Identification of a new HIV type 1 circulating BF intersubtype recombinant form (CRF47_BF) in Spain. AIDS Res. Hum. Retroviruses 26, 827-832. doi: 10.1089/aid.2009.0311

Gill, M., Lemey, P., Faria, N., Rambaut, A., Shapiro, B., and Suchard, M. (2013). Improving Bayesian population dynamics inference: a coalescent-based model for multiple loci. Mol. Biol. Evol. 30, 713-724. doi: 10.1093/molbev/mss265

Guimarães, M. L., Eyer-Silva, W. A., Couto-Fernandez, J. C., and Morgado, M. G. (2008). Identification of two new CRF_BF in Rio de Janeiro State, Brazil. AIDS 22, 433-435. doi: 10.1097/QAD.0b013e3282f47ad0

Guindon, S., Dufayard, J. F., Lefort, V., Anisimova, M., Hordijk, W., and Gascuel, O. (2010). New algorithms and methods to estimate maximum-likelihood phylogenies: assessing the performance of PhyML 3.0. Syst. Biol. 59, 307-321. doi: $10.1093 /$ sysbio/syq010

Hemelaar, J., Elangovan, R., Yun, J., Dickson-Tetteh, L., Kirtley, S., GouwsWilliams, E., et al. (2020). Global and regional epidemiology of HIV-1 recombinants in 1990-2015: a systematic review and global survey. Lancet HIV 7, e772-e781. doi: 10.1016/S1473-3099(18)30647-9

Hoang, D. T., Chernomor, O., von Haeseler, A., Minh, B. Q., and Vinh, L. S. (2018). UFBoot2: improving the ultrafast bootstrap approximation. Mol. Biol. Evol. 35, 518-522. doi: 10.1093/molbev/msx281

Instituto Nacional de Estadística (2021a). Principales Series De Población Desde 1998. Available online at: https://www.ine.es/jaxi/Datos.htm?path=/t20/e245/ p08/10/, andfile=01006.px\#!tabs-tabla (Accessed August 17, 2021).

Instituto Nacional de Estadística (2021b). Migraciones Exteriores. Available online at: https://www.ine.es/jaxiT3/Tabla.htm?t=24295 (Accessed August 17, 2021).

Instituto Nacional de Estadísticas y Censos, República Argentina (2021). Censo 2010. Available online at: https://www.indec.gob.ar/indec/web/Nivel4-Tema2-41-135 (Accessed August 17, 2021).

Kalyaanamoorthy, S., Minh, B. Q., Wong, T. K. F., von Haeseler, A., and Jermiin, L. S. (2017). ModelFinder: fast model selection for accurate phylogenetic estimates. Nat. Methods 14, 587-589. doi: 10.1038/nmeth

Katoh, K., and Standley, D. M. (2013). MAFFT multiple sequence alignment software version 7: improvements in performance and usability. Mol. Biol. Evol. 30, 772-780. doi: 10.1093/molbev/mst010
Kumar, S., Stecher, G., and Tamura, K. (2016). MEGA7: molecular evolutionary genetics analysis version 7.0 for bigger datasets. Mol. Biol. Evol. 33, 1870-1874. doi: 10.1093/molbev/msw054

Lai, A., Bozzi, G., Franzetti, M., Binda, F., Simonetti, F. R., Micheli, V., et al. (2014). Phylogenetic analysis provides evidence of interactions between Italian heterosexual and South American homosexual males as the main source of national HIV-1 subtype C epidemics. J. Med. Virol. 86, 729-736. doi: 10.1002/ jmv.23891

Lole, K. S., Bollinger, R. C., Paranjape, R. S., Gadkari, D., Kulkarni, S. S., Novak, N. G., et al. (1999). Full-length human immunodeficiency virus type 1 genomes from subtype C-infected seroconverters in India, with evidence of intersubtype recombination. J. Virol. 73, 152-160. doi: 10.1128/JVI.73.1.152-160. 1999

Los Alamos National Laboratory (2021). HIV Sequence Database. Available online at: https://www.hiv.lanl.gov/content/sequence/HIV/mainpage.html (Accessed August 17, 2021).

Louwagie, J., Delwart, E. L., Mullins, J. I., McCutchan, F. E., Eddy, G., and Burke, D. S. (1994). Genetic analysis of HIV-1 isolates from Brazil reveals presence of two distinct genetic subtypes. AIDS Res. Hum. Retroviruses 10, 561-567. doi: 10.1089/aid.1994.10.561

Nájera, R., Delgado, E., Pérez-Álvarez, L., and Thomson, M. M. (2002). Genetic recombination and its role in the development of the HIV-1 pandemic. AIDS 16, S3-S16. doi: 10.1097/00002030-200216004-00002

Pessôa, R., Watanabe, J. T., Calabria, P., Felix, A. C., Loureiro, P., Sabino, E. C., et al. (2014). Deep sequencing of HIV-1 near full-length proviral genomes identifies high rates of BF1 recombinants including two novel circulating recombinant forms (CRF) 70_BF1 and a disseminating 71_BF1 among blood donors in Pernambuco, Brazil. PLoS One 9:e112674. doi: 10.1371/journal.pone.011 2674

Pineda-Peña, A. C., Faria, N. R., Imbrechts, S., Libin, P., Abecasis, A. B., Deforche, K., et al. (2013). Automated subtyping of HIV-1 genetic sequences for clinical and surveillance purposes: performance evaluation of the new REGA version 3 and seven other tools. Infect. Genet. Evol. 19, 337-348. doi: 10.1016/j.meegid. 2013.04.032

Price, M. N., Dehal, P. S., and Arkin, A. P. (2010). FastTree 2-approximately maximum-likelihood trees for large alignments. PLoS One 5:e9490. doi: 10. 1371/journal.pone.0009490

Rambaut, A., Lam, T. T., Max, C. L., and Pybus, O. G. (2016). Exploring the temporal structure of heterochronous sequences using TempEst (formerly Path-O-Gen). Virus Evol. 2:vew007. doi: 10.1093/ve/vew007

Reis, M. N. G., Bello, G., Guimarães, M. L., and Stefani, M. M. A. (2017). Characterization of HIV-1 CRF90_BF1 and putative novel CRFs_BF1 in Central West, North and Northeast Brazilian regions. PLoS One 12:e0178578. doi: 10.1371/journal.pone.0178578

Reis, M. N. G., Guimarães, M. L., Bello, G., and Stefani, M. M. A. (2019). Identification of new HIV-1 circulating recombinant forms CRF81_cpx and CRF99_BF1 in Central Western Brazil and of Unique BF1 recombinant forms. Front. Microbiol. 10:97. doi: 10.3389/fmicb.2019.00097

Ristic, N., Zukurov, J., Alkmim, W., Diaz, R. S., Janini, L. M., and Chin, M. P. (2011). Analysis of the origin and evolutionary history of HIV-1 CRF28_BF and CRF29_BF reveals a decreasing prevalence in the AIDS epidemic of Brazil. PLoS One 6:e17485. doi: 10.1371/journal.pone.0017485

Robertson, D. L., Anderson, J. P., Bradac, J. A., Carr, J. K., Foley, B., Funkhouser, R. K., et al. (2000). HIV-1 nomenclature proposal. Science 288, 55-56. doi: $10.1126 /$ science.288.5463.55d

Ruchansky, D., Casado, C., Russi, J. C., Arbiza, J. R., and López-Galíndez, C. (2009). Identification of a new HIV type 1 circulating recombinant form (CRF38_BF1) in Uruguay. AIDS Res. Hum. Retroviruses 25, 351-356. doi: 10.1089/aid.2008. 0248

Salminen, M. O., Carr, J. K., Burke, D. S., and McCutchan, F. E. (1995). Identification of breakpoints in intergenotypic recombinants of HIV type 1 by bootscanning. AIDS Res. Hum. Retroviruses 11, 1423-1425. doi: 10.1089/aid. 1995.11.1423

Sanabani, S. S., Pastena, E. R., Neto, W. K., Martinez, V. P., and Sabino, E. C. (2010). Characterization and frequency of a newly identified HIV-1 BF1 intersubtype circulating recombinant form in São Paulo Brazil. Virol. J. 7:74. doi: 10.1186/ 1743-422X-7-74 
Schultz, A. K., Zhang, M., Bulla, I., Leitner, T., Korber, B., Morgenstern, B., et al. (2009). jpHMM: improving the reliability of recombination prediction in HIV-1. Nucleic Acids Res. 37, W647-W651. doi: 10.1093/nar/gkp371

Shapiro, B., Rambaut, A., and Drummond, A. J. (2006). Choosing appropriate substitution models for the phylogenetic analysis of protein-coding sequences. Mol. Biol. Evol. 23, 7-9. doi: 10.1093/molbev/msj021

Sierra, M., Thomson, M. M., Ríos, M., Casado, G., Ojea de Castro, R., Delgado, E., et al. (2005). The analysis of near full-length genome sequences of human immunodeficiency virus type $1 \mathrm{BF}$ intersubtype recombinant viruses from Chile, Venezuela and Spain reveals their relationship to diverse lineages of recombinant viruses related to CRF12_BF. Infect. Genet. Evol. 5, 209-217. doi: 10.1016/j.meegid.2004.07.010

Simonetti, F. R., Lai, A., Monno, L., Binda, F., Brindicci, G., Punzi, G., et al. (2014). Identification of a new HIV-1 BC circulating recombinant form (CRF60_BC) in Italian young men having sex with men. Infect. Genet. Evol. 23, 176-181. doi: 10.1016/j.meegid.2014.02.007

Struck, D., Roman, F., De Landtsheer, S., Servais, J. Y., Lambert, C., Masquelier, C., et al. (2015). Near full-length characterization and population dynamics of the human immunodeficiency virus type 1 circulating recombinant form 42 (CRF42_BF) in Luxembourg. AIDS Res. Hum. Retroviruses 31, 554-558. doi: 10.1089/AID.2014.0364

Suchard, M. A., Lemey, P., Baele, G., Ayres, D. L., Drummond, A. J., and Rambaut, A. (2018). Bayesian phylogenetic and phylodynamic data integration using BEAST 1.10. Virus Evol. 4:vey016. doi: 10.1093/ve/vey016

Thomson, M. M., and Nájera, R. (2005). Molecular epidemiology of HIV-1 variants in the global AIDS pandemic: an update. AIDS Rev. 7, 210-224.

Thomson, M. M., Fernández-García, A., Delgado, E., Vega, Y., Díez-Fuertes, F., Sánchez-Martínez, M., et al. (2012). Rapid expansion of a HIV-1 subtype F cluster of recent origin among men who have sex with men in Galicia, Spain. J. Acquir. Immune Defic. Syndr. 59, e49-e51. doi: 10.1097/QAI. 0b013e3182400fc4

Thomson, M. M., Herrero, I., Villahermosa, M. L., Vázquez de Parga, E., Cuevas, M. T., Carmona, R., et al. (2002). Diversity of mosaic structures and common ancestry of human immunodeficiency virus type $1 \mathrm{BF}$ intersubtype recombinant viruses from Argentina revealed by analysis of near full-length genome sequences. J. Gen. Virol. 83, 107-119. doi: 10.1099/0022-1317-83-1-107
Thomson, M. M., Villahermosa, M. L., Vázquez de Parga, E., Cuevas, M. T., Delgado, E., Manjón, N., et al. (2000). Widespread circulation of a B/F intersubtype recombinant form among HIV-1-infected individuals in Buenos Aires, Argentina. AIDS 14, 897-899. doi: 10.1097/00002030-20000505000020

Trifinopoulos, J., Nguyen, L. T., von Haeseler, A., and Minh, B. Q. (2016). W-IQTREE: a fast online phylogenetic tool for maximum likelihood analysis. Nucleic Acids Res. 44, W232-W235. doi: 10.1093/nar/gkw256

Vinken, L., Fransen, K., Cuypers, L., Alexiev, I., Balotta, C., Debaisieux, L., et al. (2019). Earlier initiation of antiretroviral treatment coincides with an initial control of the HIV-1 sub-subtype F1 outbreak among men-having-sex-withmen in Flanders, Belgium. Front. Microbiol. 10:613. doi: 10.3389/fmicb.2019. 00613

Zhang, M., Foley, B., Schultz, A. K., Macke, J. P., Bulla, I., Stanke, M., et al. (2010). The role of recombination in the emergence of a complex and dynamic HIV epidemic. Retrovirology 7:25. doi: 10.1186/1742-4690-7-25

Conflict of Interest: The authors declare that the research was conducted in the absence of any commercial or financial relationships that could be construed as a potential conflict of interest.

Publisher's Note: All claims expressed in this article are solely those of the authors and do not necessarily represent those of their affiliated organizations, or those of the publisher, the editors and the reviewers. Any product that may be evaluated in this article, or claim that may be made by its manufacturer, is not guaranteed or endorsed by the publisher.

Copyright (C) 2021 Bacqué, Delgado, Benito, Moreno-Lorenzo, Montero, Gil, Sánchez, Nieto-Toboso, Muñoz, Zubero-Sulibarria, Ugalde, García-Bodas, Cañada, del Romero, Rodríguez, Rodríguez-Avial, Elorduy-Otazua, Portu, García-Costa, Ocampo, Cabrera and Thomson. This is an open-access article distributed under the terms of the Creative Commons Attribution License (CC BY). The use, distribution or reproduction in other forums is permitted, provided the original author(s) and the copyright owner(s) are credited and that the original publication in this journal is cited, in accordance with accepted academic practice. No use, distribution or reproduction is permitted which does not comply with these terms. 